\title{
Chemopreventive Effects of Cocoa Extract Application Towards Expression of Bcl-2 and Mda on Albino Mice Skin Induced by Dmba-Tpa
}

\author{
Khairuddin Djawad, Munawar Ambar, Siswanto Wahab \\ Department of Dermatology and Venereology, Medical Faculty, Hasanuddin University, Makassar, Indonesia
}

Email address:

ambar_erie@yahoo.com (M. Ambar)

To cite this article:

Khairuddin Djawad, Munawar Ambar, Siswanto Wahab. Chemopreventive Effects of Cocoa Extract Application Towards Expression of Bcl2 and Mda on Albino Mice Skin Induced by Dmba-Tpa. American Journal of Clinical and Experimental Medicine.

Vol. 5, No. 3, 2017, pp. 97-101. doi: 10.11648/j.ajcem.20170503.16

Received: March 21, 2017; Accepted: March 31, 2017; Published: May 18, 2017

\begin{abstract}
This research aims to determine the effect of topical cocoa extract application through ekspression of Bcl-2 and MDA on albino mice skin that induced by DMBA-TPA. The research was conducted at Animal Laboratory for application of DMBA, MDA-TPA and cocoa extract and Biomolecular Laboratory of Medical Faculty of Hasanuddin University Makassar as the place of ELISA examination. The method used was animal experimental with pure experimental design to know the role of topical cocoa extract towards the skin of mice which has been given DMBA-TPA three times a week in 12 weeks. The research unit was the skin of the back side of albino mice which have been excised and divided into 5 groups of treatment. The results indicate that extract of concentration cocoa $400 \mathrm{ppm}(\mathrm{p}<0.05)$ which is applied every day has the highest effects decreased of Bcl-2 and MDA expression after being inducted by DMBA-TPA three times a week.
\end{abstract}

Keywords: Bcl-2, Cacao Extract, DMBA, Malondialdehyde (MDA), TPA

\section{Introduction}

Bcl2 family proteins regulate apoptosis along the intrinsic mitochondrial apoptosis pathway that is activated in response to a number of stress stimuli including growth-factor deprivation, cytokine-withdrawal, $\mathrm{Ca}^{++}$-flux or DNAdamage, caused by UV or gamma- irradiation, but can also be licensed to contribute to cell death triggered by members of the tumour necrosis factor family member such as Fas, TNF or TRAIL. Based on their function, the members of the Bcl2 family can be divided into pro-apoptotic and prosurvival proteins. Pro-survival proteins contain up to four Bcl2 homology (BH) domains. The BH1, 2 and 3 domains are critically involved in the creation of a hydrophobic groove where the $\alpha$-helix formed by the BH3-domain of a pro-apoptotic partner is binding into The molecular function of the BH4-domain, although required for the anti-apoptotic function of pro-survival Bcl2-family proteins still remains to be fully elucidated. Pro- apoptotic Bcl2 family members can be subdivided into multi-domain class proteins, harbouring three out of four BH-domains (BH1, 2, 3) i.e. Bak, Bax and
Bok and those that only contain the BH3-domain and are referred to as $\mathrm{BH} 3$-only proteins, including $\mathrm{Bim} / \mathrm{Bod}$, Bid, Bmf, Puma/Bbc3, Noxa/APR, Bad, Bik/Nbk/Blk and Hrk/DP5.

Malondialdehyde (MDA) is an endogenous genotoxic product of enzymatic and oxygen radical-induced lipid peroxidation whose adducts are known to exist in DNA isolated from healthy human beings. To evaluate the mutagenic potential of MDA in human cells, we reacted MDA with pSP189 shuttle vector DNA and then transfected them into human fibroblasts for replication. MDA induced up to a 15 -fold increase in mutation frequency in the supF reporter gene compared with untreated DNA. Sequence analysis revealed that the majority of MDA-induced mutations occurred at GC base pairs. The most frequent mutations were large insertions and deletions, but base pair substitutions were also detected. MDA-induced mutations were completely abolished when the adducted shuttle vector was replicated in cells lacking nucleotide excision repair. 
MDA induction of large deletions and the apparent requirement for nucleotide excision repair suggested the possible involvement of a DNA interstrand cross-link as a premutagenic lesion. Indeed, MDA formed interstrand crosslinks in duplex plasmids and oligonucleotides. Substrates containing the sequence 5 '-d (CG) were preferentially crosslinked, consistent with the observation of base pair substitutions in $5^{\prime}$-d (CG) sites in the MDA-induced mutation spectrum. These experiments provide biological and biochemical evidence for the existence of MDA-induced DNA interstrand cross-links that could result from endogenous oxidative stress and likely have potent biological effects. [1]

The compound 7, 12-dimetilbenz ( $\alpha$ ) antrasen (DMBA) is a chemical that is included in the polycyclic aromatic hydrocarbons (PAH), which are known to be mutagenic, teratogenic, carcinogenic, cytotoxic and immunosuppressive. According to the Division of Occupational Health and Safety National Institutes of Health, which has DMBA 4 benzene rings included in the seven PHAs can cause cancer in humans. Naturally DMBA can be found in nature as a result of incomplete combustion processes, such as tobacco smoke, wood burning smoke, smoke burning gas, gasoline, oil, coal or meat. [2]. The compound 7, 12-Dimethylbenz [ $\alpha]$ anthracene is an immune suppressant and organ-specific carcinogens powerful laboratory. Also known as 7, 12dimethylbenz $[\alpha]$ anthracene or DMBA, this substance is widely used in research laboratories studying cancer. Serves as a tumor initiator DMBA to make cell mutation. Tumor promotion can be induced by treatment with TPA (12-Otetradecanoylphorbol-13-acetate) in two stages of carcinogenesis. This enables the acceleration of tumor growth, so that research on many cancers do. [3]

Heinrich et al found that oral consumption of cocoa in the long term has a beneficial effect as photoprotection against UV radiation that increases blood flow to the dermis dan thickened skin, increased skin density and humidity that affect the skin structure. [4] Othman A et al (2007) conducted research on the effectiveness of cocoa extract which shows cocoa extract has a higher antioxidant effect than ethanol. Antioxidant components in the extract consists of amino acids, phenolic acid, flavonoids. [5]

Although the benefits of cocoa as antioxidant has been known in many diseases, but the chemopreventive effect of topical application in various concentrations to prevent the carcinogenesis process as an antioxidant so far unknown, especially the protective effects of cocoa extract by assessing the expression of MDA and Bcl-2 on albino mice skin induced DMBA/TPA for 12 weeks.

\section{Material and Methods}

\subsection{Location and Time Research}

The study was conducted at the Animal Laboratory for application of DMBA-TPA and cocoa extract and Laboratory of Biomolecular Medical Faculty of Hasanuddin University

\section{Makassar for ELISA examination.}

\subsection{Design Research}

This research was an experimental animal with a pure experimental designs.

\subsection{Population and Sample}

The population was albino mice aged 16-20 weeks with an average weight of 20-30 grams, which was obtained from the Laboratory of Balitbang Veternir Maros. Mice was maintained for 1 week under standard conditions (temperature $28 \pm 20^{\circ} \mathrm{C}$ ), humidity $50 \pm 100^{\circ} \mathrm{C}$ and the room lights with a cycle of 12 hours.

Flavonoid level contained in cocoa extract had tested respectively about $29.68 \mu \mathrm{g}$ in $400 \mathrm{ppm}$ cocoa extract solution and $59.36 \mu \mathrm{g}$ in $800 \mathrm{ppm}$ cocoa extract solution.

\subsection{Method of Collecting Data}

Each mice with inclusion criteria taken photos for the initial data and during the study. After adaptation for 1 week, 25 mice were divided into 5 groups. Group I was untreated group. Group II was DMBA-TPA control group. Group III was aceton- DMBA-TPA control group. Group IV applied with aceton, DMBA-TPA, and 400 ppm cocoa extract solution. Group V applied with aceton, DMBA-TPA, and 800 ppm cocoa extract solution. Control and treated groups had induced by DMBA three times a week for 1 week, continued with application TPA three timea a week for 11 weeks. The body weight was measured every week. All mices were terminated using ether and performed a $2 \times 2 \mathrm{~cm}$ excisional biopsy on the middle back skin of mice and stored in Nacl $0.9 \%$ solution for ELISA examination.

\subsection{Statistical Analysis}

All data collected obtained from the results of subsequent studies were edited, tabulations and entered into a SPSS program. Data from 5 groups will be tested normality with $\mathrm{T}$ test (Mann-Whitney Test). If the distribution of normal data, then tested by ANOVA followed by Post Hoc test test in statistical programme for socials sciences (SPSS) version 22.0. with significancy value if $\mathrm{p}<0.05$.

\section{Results}

Elisa examination showed MDA expression in DMBATPA control group different significantly with the untreated group. $400 \mathrm{ppm}$ cocoa group was different significantly to the untreated group and did not different significantly with DMBA-TPA control group. Cocoa 800 ppm group was not different significantly with DMBA-TPA control group. As shown in Table 1.

All treatment groups showed that expression of Bcl-2 in 400 ppm cocoa group lower than 800 ppm cocoa group. Bcl2 expression in $400 \mathrm{ppm}$ cocoa group different significantly with DMBA-TPA control group and did not different significantly with the untreated group. As shown in table 2 . 
Table 1. Malondialdehyde expression after application with DMBA-TPA and cocoa extract $400 \mathrm{ppm}$ and $800 \mathrm{ppm}$.

\begin{tabular}{llll}
\hline Treated Group & N & Mean & P \\
\hline Untreated group & 5 & $1,165 \pm 1,338$ & $0,07^{\mathrm{a}}$ \\
DMBA & 5 & $6,910 \pm 1,116$ & $0,00^{\mathrm{b}}$ \\
Aceton+DMBA & 5 & $6,862 \pm 1,523$ & $0,97^{\mathrm{c}}$ \\
Aceton+DMBA+cocoa $400 \mathrm{ppm}$ & 5 & $3,620 \pm 2,359$ & $0,02^{\mathrm{d}}$ \\
Aceton+DMBA+cocoa $400 \mathrm{ppm}$ & 5 & $4,842 \pm 3,188$ & $0,12^{\mathrm{e}}$ \\
\hline
\end{tabular}

Note: Significans; a between untreated group and cocoa 400 ppm group, b, between DMBA and and untreated group, c; between DMBA and aceton group, d, between DMBA and cocoa 400 ppm group, and e, between DMBA and cocoa 800 ppm group

Table 2. Bcl-2 expression after treating with DMBA and cocoa 400 ppm and $800 \mathrm{ppm}$.

\begin{tabular}{llll}
\hline Treated Group & N & Mean & P \\
\hline Untreated group & 5 & $24,3 \pm 13,153$ & $0,09^{\mathrm{a}}$ \\
DMBA & 5 & $357,61 \pm 197$ & $0,01^{\mathrm{b}}$ \\
Aceton+DMBA & 5 & $333,648 \pm 204$ & $0,77^{\mathrm{c}}$ \\
Aceton+DMBA+cocoa 400 ppm & 5 & $175,019 \pm 35$ & $0,04^{\mathrm{d}}$ \\
Aceton+DMBA+cocoa 400 ppm & 5 & $2227,68 \pm 84$ & $0,14^{\mathrm{e}}$ \\
\hline
\end{tabular}

Note: Significans; a between untreated group and cocoa 400 ppm group, $b$, between DMBA and and untreated group, c; between DMBA and aceton group, d, between DMBA and cocoa 400 ppm group, and e, between DMBA and cocoa 800 ppm group

\section{Discussion}

Cocoa extract was using as protective material in this study based on high falvonoid content. Cocoa contains epicatechin and catechin fraction of flavonoid and procyanidin. The content of easily absorbed. The mechanism of action of various studies extract cocoa flavonoid component is still under study, but based on proven research of flavonoids as antioxidants interact with intracellular signaling to get a variety of biological effects. In this research flavonoid level contained in cocoa extract had tested respectively about $29.68 \mu \mathrm{g}$ in $400 \mathrm{ppm}$ cocoa extract solution and $59.36 \mu \mathrm{g}$ in $800 \mathrm{ppm}$ cocoa extract solution. Cocoa therapy prior to induction of oxidative stress preventing DNA damage and increase the activity of antioxidant enzyme glutathione reductase and glutathione peroxidase in cells induced by prooxidant: tetrabuthylhydroperoxyde. Based on this study also used cacao therapy before induction of pro-oxidants in mice.

\subsection{DMBA-TPA Application Effect at Various Treatment Groups on Levels of MDA}

Table 1 showed that application cocoa prior to application prooxidative DMBA-TPA shown to inhibit oxidative stress with the best protective effects at concentrations $400 \mathrm{ppm}$.

Oxidative stress is generally found in cell membranes as MDA resulting from the process of enzymatic oxidation and lipid peroxidation. Various studies show that MDA will induce mutations that cause cancer. [6] This study uses the measurements to determine the level of MDA-induced oxidative stress in mice by DMBA-TPA applications. ELISA results showed DMBA/TPA-induced oxidative stress in the DMBA control group whose levels are significantly higher than the negative control group. When compared with the protective group of cocoa, MDA expression of cocoa 400 ppm group significantly lower than the control group, provided that cocoa can inhibit the formation of oxidative stress. ANOVA test results showed group of cocoa $800 \mathrm{ppm}$ had no significant difference with DMBA control group so $400 \mathrm{ppm}$ cocoa group is the best protective group even statitstic test results showed no significant difference with the untreated group. Anti-oxidant effects of cocoa is consistent with research Romiro IR 2011, proved on experimental research that the cocoa extract can inhibit oxidative stress induced by the application of heat to the winstar rat leukocytes. Other studies in vitro prove cocoa extract antioxidant effect on biomolecular research STAT1 gene, MAPKK, MRP1 is associated with oxidative stress. [7, 8] Effects of cocoa extract has also been demonstrated to prevent overproduction of oxidative stress and prevent the reduction of antioxidant enzymes gluthation, in other studies that use induction prooxidative tetrabutylhydroperoxide HepG2 cells. [9]

Zhu prove in vitro studies cocoa extract antioxidant effects that are dose dependent on oxidative stress induced by erythrocyte hemolysis. [10] Counet prove epicatechin is the primary unit among the various units of flavanols in flavonoids which act as anti-oxidants. [11]

\subsection{DMBA-TPA Application Effect at Various Treatment Groups on the Expression of Bcl-2}

Tabel 2 showed Bcl-2 expression in DMBA-TPA control group higher significant than untreated control group, which indicates the application DMBA/TPA can induce Bcl-2 expression, that was being compared to the treatment group that applied the extract of cocoa. Results of statistical analysis showed a group of $400 \mathrm{ppm}$ cocoa had a preventive effect on the expression of Bcl-2 better than cocoa group of $800 \mathrm{ppm}$. Consistent with previous studies that cocoa has a proapoptotic effect via down regulation of $\mathrm{Bcl}-2$. [12] Proapoptotic effect is determined by the positive ratio between BAX and Bcl-2, then induces the release of cytochrome $\mathrm{C}$ from mitochondria which is the determinant of apoptosis. [13]

Applications DMBA shown to induce free radicals that damage DNA. The effects of such damage can induce apoptosis or mutate if the damage can not be repaired. Arlio suggests that induction of apoptosis through increased gene expression of Bax and decreased Bcl-2. [14] Other studies prove cacao extract induces proapoptosis BAX gene, inhibits the $\mathrm{Bcl}-2$ gene and activation of caspase-3 in the process of apoptosis. [15]

From the table 2 the results showed $\mathrm{Bcl}-2$ expression of DMBA control group significantly higher in the untreated control group being compared to the treatment group that applied the extract of cocoa which indicates the application DMBA can induce Bcl-2. Statistical analysis showed cocoa $400 \mathrm{ppm}$ group proved to have a preventive effect on the expression of $\mathrm{Bcl}-2$ better than cocoa $800 \mathrm{ppm}$ group Consistent with previous studies that cocoa has a 
proapoptotic effect via down regulation of Bcl-2. [12] Proapoptotic effect is determined by the positive ratio between $\mathrm{BAX}$ and $\mathrm{Bcl}-2$, then induces the release of cytochrome $\mathrm{C}$ from mitochondria which is the determinant of apoptosis. [13] Anti-oxidant effects of cocoa extract in this study according to the results of Elisa examination that showed $400 \mathrm{ppm}$ is the best treatment group as antioxidants and prevent increase expression of $\mathrm{Bcl}-2$. This is consistent with other studies that showed low expression of Bcl-2 which trigger apoptosis.

Increasing concentrations of cocoa statistically no effect on levels of Bcl-2 were not statistically significantly different but there is a trajectory in line with the effects of increasing concentrations of cocoa. Interestingly the more effective an antioxidant, the cytotoxic and antiproliferative they are. This is caused by the dual effects of antioxidant/prooxidant of polyphenols or also in their capacity as a radical scavenger or yield depending on the environment.

This is consistent with the study by Martin et al (2008) shows the initial therapy cocoa extract before application prooxidative tetrabutylhydroperoxide in HepG2 liver cells are proven to prevent over-production of oxidative stress and prevent the reduction of antioxidant enzymes gluthation. Applications DMBA shown to induce free radicals that damage DNA. The effects of such damage can induce apoptosis or mutate if the damage can not be repaired. Arlio suggests that induction of apoptosis through increased expression of Bax gene and decreased Bcl-2. [14] Other studies prove cocoa extract induces proapoptosis BAX gene, inhibits the Bcl-2 gene and activation of caspase- 3 in the process of apoptosis. [15]

Studies show that consumption of oral and topical applications of polyphenols before UVB radiation to protect skin from the development of skin cancer in mice induced by UVB radiation. Polyphenols have a photoprotective effect against UV-induced skin inflammation, proliferation, immunosuppression, DNA damage and dysregulation of multiple cellular signaling pathways that are important and implications for the treatment of cancer. [16]

This study shows that cocoa extract concentration of 400 ppm $(\mathrm{p}<0.05)$ were applied every day has the highest effect on inhibiting decrease the levels of Bcl-2 and MDA after induction of DMBA three times a week.

The results showed the levels of Bcl-2 and MDA groups of applications every day higher than the control group so that application of DMBA in this group proved to induce Bcl-2 and MDA. Results of statistical analysis of the treatment groups showed significance $(\mathrm{p}<0.05)$ so that the protective cocoa group is proven statistically inhibit $\mathrm{Bcl}-2$ and MDA levels. Consistent with previous studies that cocoa has a proapoptotic effect via down regulation of Bcl-2. [12] Proapoptotic effect is determined by the positive ratio between $\mathrm{BAX}$ and $\mathrm{Bcl}-2$, then induces the release of cytochrome $\mathrm{C}$ from mitochondria which is a determinant of apoptosis.

\section{Conclusion}

In conclusion, we have demonstrated that expresions of Bcl-2 and MDA decreased with application of cocoa 400 ppm every day after induced by DMBA-TPA application three times a week. Chemopreventive effect of application topical cocoa based on a high flavonoid content. Cocoa contains epicatechin and catechin fraction of flavonoid and procyanidin to induction of oxidative stress preventing DNA damage and increase the activity of antioxidant enzyme glutathione reductase and glutathione peroxidase in cells induced by prooxidant.

\section{References}

[1] Niedernhofer LJ, Daniels Js, Rouzer CA et. al. Malondialdehyde, a Product of Lipid Peroxidation, is Mutagenic in Human Cells. JBC 278 (33): p. 31426-33.

[2] Muqbil I, Azmi AS, Banu N. Prior exposure to restraint stress enhances 7, 12-dimethylbenz (a) anthracene (DMBA) induced DNA damage in rats, 2006. FEBS Letters 580 (2): p. 39953999.

[3] Lukitaningsih E, Noegrohati S.. Studi pemisahan senyawa hidrokarbon poliaromatik secara kromatografi gas kolom kapiler. MFI. 2000. 11 (1): p. 31-38.

[4] Gasser P, Lati E, Peno-Mazzarino L, Bouzoud D, Allegaert L, Bernaert H., Cocoa polyphenolls and their influence on parameters involved in ex vivo skin restructuring. Int $\mathrm{J}$ Cosmet Sci. 2008. 30 (5): p. 339-45.

[5] Othman A, Ismail A, Ghani NA, Adenan I, et al. Antioxidant capacity and phenolic content of cocoa beans. Food Chemistry.100: p. 1523-30.

[6] Cooke MS et al., Oxidative DNA damage: mechanism, mutation, and disease. FASEB J. 2003. 17: p. 1195-1214.

[7] Nigam and Shukla,. Preventive Effects of Diallyl Sulfida on 7, 12 dimetilbenz (a) antrasen (DMBA) in Tumor Skin, Mol Nuir Food Res, 2007. 51: p. 1324-26.

[8] Romiro IR, Ramos S, Oliva EL, et al., Cocoa-rich diet prevents azoxymethane-induced colonic preneoplastic lesions in rats by restraining oxidative stress and cell proliferation and inducing apoptosis. Mol Nutr; 2011. 55 (12): p. 1895-9.

[9] Marthin MA, Serrano ABG, Ramos S et al. Protection of Human HepG2 Cells against Oxidative Stress by Cocoa Phenolic Extract. J. Agric. Food Chem, 2008. 56: p. 776572.

[10] Zhu QY, Schramm DD, Gross HB, Holt RR, Kim SH, et al., Influence of cocoa flavanols and procyanidins on free radicalinduced human erythrocyte hemolysis. Clinical \& Developmental Immunology, 2005; 12 (1): p. 27-34.

[11] Grigorof B., Reactive Oxygen Species and Their Relation to Carcinogenesis. Trakia Journal of Sciences. 2012. 10 (3): p. 83-92.

[12] Andujar I, Recio MC, Giner RM, Rios JL. Cocoa polyphenols and their potential benefits for human health. Oxidative medicine and cellular longevity, 2012, p. 1-24. 
[13] Ponten F, Berg C, Ahmadian A. Molecular pathology in basal cell cancer with p53 as genetic marker. Oncogen. 1997; 15: p. 1059-67.

[14] Butel JS., Viral Carcinogenesis Revelation of Molecular Mechanism and Etiology of Human Disease. Carcinogenesis. 2000. 21: p. 405.
[15] Bickers DR and Athar M., Oxidative Stress in the Pathogenesis of Skin Diseases. JID. 2006. 126: p. 2565-75.

[16] Afaq F, Katiyar SK, Polyphenols: Skin Photoprotection and Inhibition of Photocarcinogenesis. Mini Rev Med Chem. 2011 11 (14): p. 1200-15. 